Conf- $950793--8$

UCRL-JC-119323

PREPRINT

\title{
Filtered Fluorescer X-ray Detector
}

\author{
H. Carl Bruns \\ James A. Emig \\ Robert S. Thoe \\ Paul T. Springer \\ John A. Hernandez
}

This paper was prepared for the 1995 Annual International

Symposium on Optical Science, Engineering and Instrumentation

San Diego, CA, July 9-14,1995

April 1995

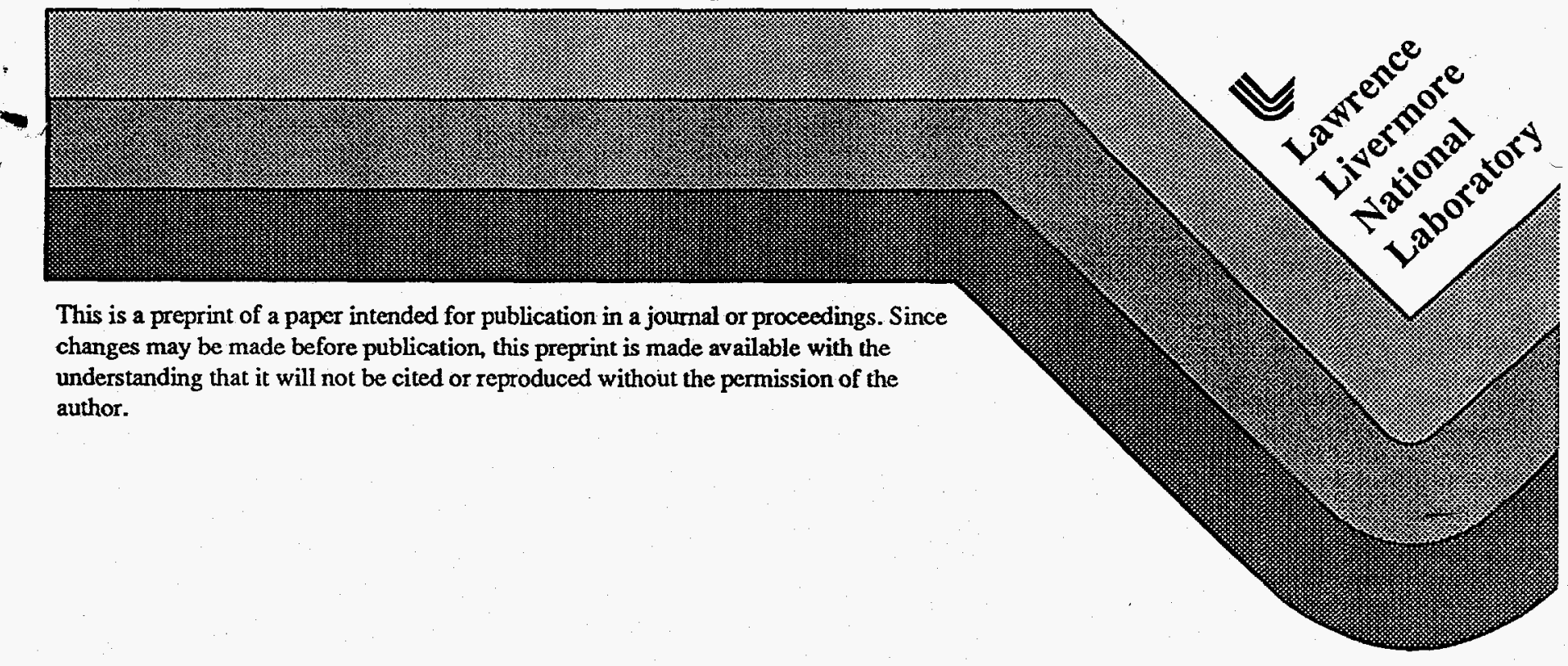




\section{DISCLAIMER}

This document was prepared as an account of work sponsored by an agency of the United States Government. Neither the United States Government nor the University of California nor any of their employees, makes any warranty, express or implied, or assumes any legal hiability or responsibility for the accuracy, completeness, or usefulness of any information, apparatus, product, or process disclosed, or represents that its use would not infringe privately owned rights. Reference herein to any specific commercial products, process, or service by trade name, trademark, manufacturer, or otherwise, does not necessarily constitute or imply its endorsement, recommendation, or favoring by the United States Government or the University of California. The views and opinions of authors expressed herein do not necessarily state or reflect those of the United States Government or the University of California, and shall not be used for advertising or product endorsement purposes. 


\section{DISCLAIMER}

Portions of this document may be illegible in electronic image products. Images are produced from the best available original document. 
Filtered fluorescer $\mathrm{x}$-ray detector

H. Carl Bruns, James A. Emig, Robert S. Thoe, Paul T. Springer, John A. Hernandez

Lawrence Livermore National Laboratory

P.O. Box 808 Livermore, California 94550

\begin{abstract}
Recently, an instrument capable of measuring $\mathrm{x}$-rays between 8 and $90 \mathrm{keV}$ was conceived to help understand conditions pertaining to pulsed power research. This resulted in the development of a versatile device that would incrementally detect $x$ rays emitted at pre-determined energy bands over this range. To accomplish this, an array of well characterized filter-fluorescer combinations were produced which would allow fluoresced $\mathrm{x}$-rays to be observed by time resolved electro-optical devices. As many as sixteen channels could be utilized with each channel having a corresponding background channel. Upon completion of the device, a three week series of experiments was then successfully carried out.
\end{abstract}

\title{
1. INTRODUCTION
}

As a result of the Nuclear Test Program at Lawrence Livermore National Laboratory, the ability to measure x-ray radiation over a broad spectrum has been developed. Expertise and instruments developed to perform these measurements have found uses in laboratory situations that have similar requirements and complications as those experienced in underground experiments. Recently, a portion of this expertise and "mothballed" equipment have been resurrected to perform a series of experiments at the Saturn facility at Sandia National Laboratory in Albuquerque NM. The intent of these experiments was to characterize conditions produced by "non thermal loads" (or in this situation a variety of "wire arrays") when subjected to a Z-pinch. The anticipated power to be measured was several kilo Joules with the spectral region of interest to be $9 \mathrm{keV}$ to $90 \mathrm{keV}:{ }^{1} \mathrm{~A}$ highly sensitive instrument with the ability to reject large backgrounds was needed and therefore resulted in the device to be described. The technique to use filter and fluorescer combinations, electrostatically focusing microchannel plate (mcp) detectors, and streak camera recording equipment was deployed to address the requirements. The development and use of the filter/fluorescer assembly, and the mcp detector with it's fiber optic relay will be the focus of this paper.

\section{CONCEPTUAL DESIGN}

The technique to use combinations of filters and fluorescers to aid in selectively diagnosing x-rays levels at specific energy bands is an excellent method to perform this type of experiment for several reasons. The greatest benefit is the provision for a background channel to be used in conjunction with each data channel. In this instrument, each data channel is comprised of a "fluorescer" made of an element that has an $\mathrm{x}$-ray absorption edge near the energy to be measured. Preceding the fluorescer is an absorbing filter which has an edge just below the desired energy which allows all $\mathrm{x}$-rays above that edge to transmit to the fluorescer. X-rays at energies above the fluorescer absorption edge transmit through fluorescer while those below generate secondary $\mathrm{x}$-rays which can be observed by a detector. Between the fluorescer and detector is another filter comprised of a lower $\mathrm{Z}$ element which prevents lower energy $\mathrm{x}$-rays from reaching the detector. The corresponding background channel is nearly identical with the exception that the pre filter would be of the same element as the fluorescer, thus allowing only radiation considered to be background to be fluoresced. This background channel can then be subtracted from the data channel to determine the net $\mathrm{x}$-ray flux. Figure 1. shows this concept graphically.

figure 1.

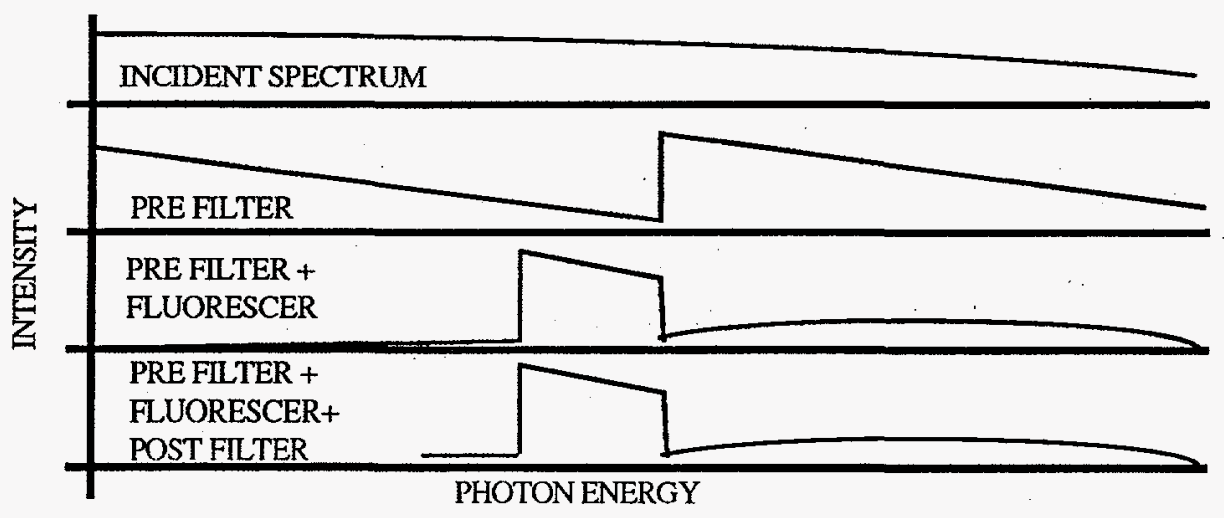


Another advantage of this device is that it allows the placement of a sensitive detector out of direct line of site of the incident beam. The detector can then be shielded as necessary from the extremely high levels of hard $\mathrm{x}$-rays produced by this facility. In this instrument a highly sensitive micro channel plate intensified detector was used that had additional sensitivity due to a two dimensionally focusing electron focusing grid.

Taking into consideration the aforementioned characteristics and the physical dimensions of both the event to be diagnosed and the Saturn chamber itself, the mechanical design was then initiated. Two of the main factors determining how the instrument would be built were the anticipated size of the pinch, and the size constraints of the detector. Since most components for the detector to be used already existed, much of the design was based on it's features. It was determined that the maximum number of channels that could be accommodated per detector would be sixteen, or four rows of four, each spaced 0.5 inch center to center. This would also determine the size and pattern of the fluorescers, filters, and baffles. Assuming the size of the event to be observed to be two centimeters in diameter, collimators and baffles could also be designed.

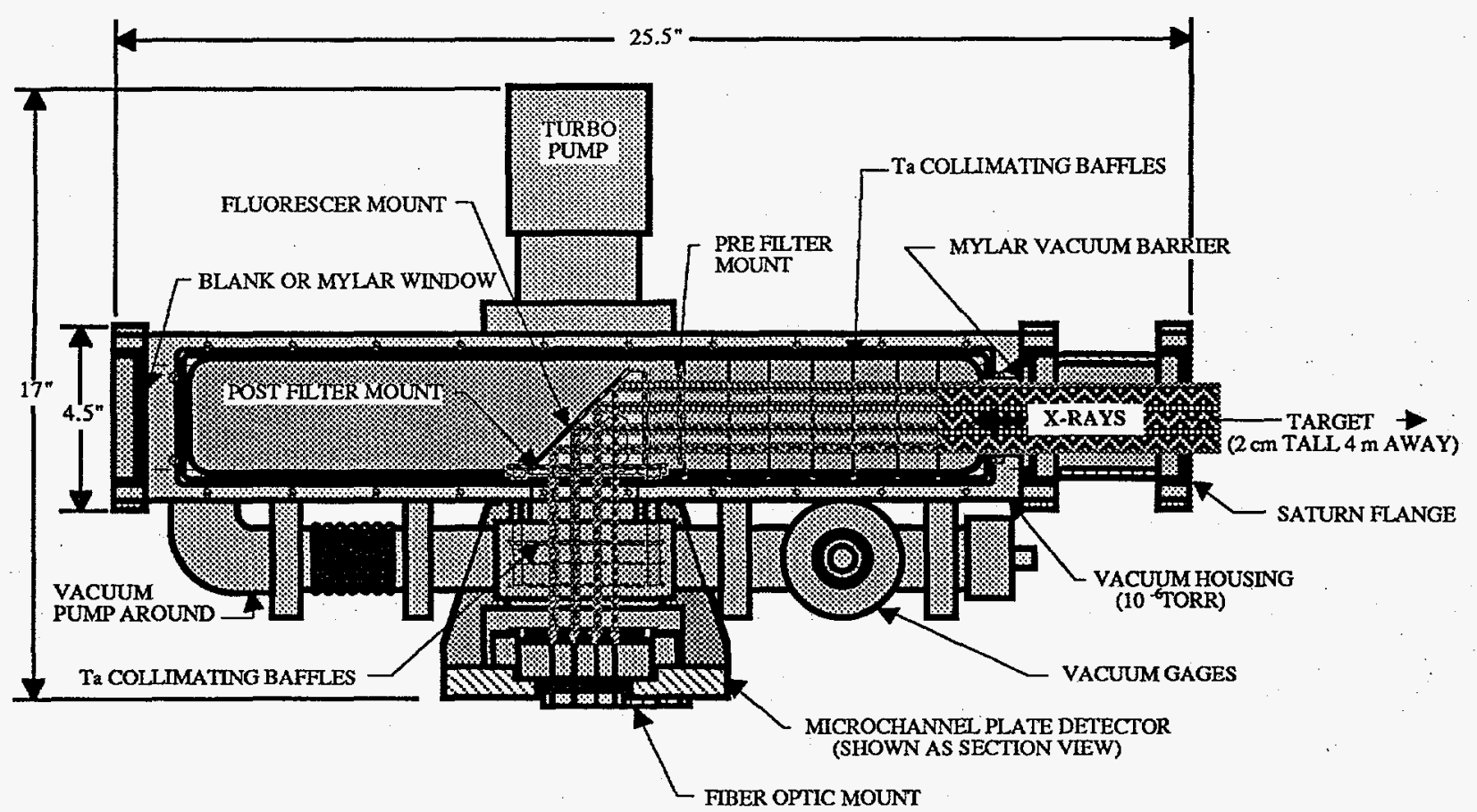

figure 2.

\section{FILTER AND FLUORESCER COMBINATIONS}

Due to the limit of sixteen channels per unit, it was decided that two assemblies would be used, one covering the lower spectral range from 9 to $45 \mathrm{keV}$ and the other covering the higher spectral range from 47 to $88 \mathrm{keV}$. With the need for each channel to have a corresponding background channel, a total of sixteen unique energy channels resulted from the two units. The specific energies measured within each range were determined by the selection of elements for each fluorescer as described above. The pre filter or absorber for each channel was selected to absorb all incident $\mathrm{x}$-rays several keV below the fluorescer edge or up to the edge for the background channels. The final absorbing filters were simply low $\mathrm{Z}$ materials transparent to the intended signal. These were typically sheets of beryllium or vanadium as opposed to individual pellets. Of course the thicknesses of all of the filters and fluorescers were important and therefore carefully calculated for in the design. Table 1. displays a list of the combinations used. 
LOW ENERGY CHANNELS

$9-42 \mathrm{keV}$

\section{HIGH ENERGY CHANNELS \\ $42-88 \mathrm{keV}$}

$\begin{array}{cccc} & \begin{array}{c}\text { fluorescer/absorber } \\ \text { flr thickness/abs thickness }\end{array} & \begin{array}{c}\text { (inches X .001) } \\ \mathrm{Mr} / \mathrm{Sm}\end{array} \\ \mathrm{Cu} / \mathrm{Ge} & \mathrm{Mo} / \mathrm{Ag} & 3.3 / 11.2 & \mathrm{Yb} / \mathrm{TA} \\ 0.5 / 1.92 & 1.0 / 3.38 & \mathrm{Pr} / \mathrm{Pr} & 9.0 / 9.5 \\ \mathrm{Cu} / \mathrm{Cu} & \mathrm{Mo} / \mathrm{Mo} & 3.3 / 14.0 & \mathrm{Yb} / \mathrm{Yb} \\ 0.5 / 1.45 & 1 / 4.62 & \mathrm{Sm} / \mathrm{Gd} & 9.0 / 24.4 \\ \mathrm{Ge} / \mathrm{Br} & \mathrm{Ag} / \mathrm{Sn} & 5.29 / 12 . & \mathrm{TA} / \mathrm{Os} \\ 0.5 / 4.42 & 1.5 / 5.65 & \mathrm{Sm} / \mathrm{Sm} & 4.4 / 7.77 \\ \mathrm{Ge} / \mathrm{Ge} & \mathrm{Ag} / \mathrm{Ag} & 5.29,14 & \mathrm{Ta} / \mathrm{Ta} \\ 0.5 / 3.25 & 1.5 / 4.42 & \mathrm{Gd} / \mathrm{Ho} & 4.4 / 11.9 \\ \mathrm{Br} / \mathrm{Y} & \mathrm{Sn} / \mathrm{I} & 5.58 / 14.7 & \mathrm{Os} / \mathrm{Au} \\ 1.5 / 4.51 & 2.5 / 10.0 & \mathrm{Gd} / \mathrm{Gd} & 3.62 / 11.12 \\ \mathrm{Br} / \mathrm{Br} & \mathrm{Sn} / \mathrm{Sn} & 5.58 / 14.7 & \mathrm{Os} / \mathrm{Os} \\ 1.5 / 8.71 & 2.5 / 7.73 & \mathrm{Ho} / \mathrm{Yb} & 3.62 / 10.0 \\ \mathrm{Y} / \mathrm{Mo} & \mathrm{Mo} & 5.92 / 19.42 & \mathrm{Au} / \mathrm{Pb} \\ 1.5 / 2.5 & 3.3 / 10.8 & \mathrm{Ho} / \mathrm{Ho} & 5.18 / 21.61 \\ \mathrm{Y} / \mathrm{Y} & \mathrm{I} / \mathrm{Au} & 5.92,16.5 & 5.18 / 13.6 \\ 1.5 / 1.08 & 3.3 / 18.7 & & \end{array}$

table 1.

\section{FABRICATION OF FILTERS AND FLUORESCERS}

As specified in table 1. a wide variety of elements were required to cover the broad spectral range. Many of the materials were readily available as sheet material while others were more difficult to acquire given the purity and thickness required. To overcome this problem a process was developed to create filters and fluorescers of the exact thickness needed. It was discovered that most of the elements required were available in the form of powders or small granules and therefore could be pressed into discs or "pellets" of a calculated density which allowed the manufacture of nearly any filter or fluorescer. Specifically, a twenty ton hydraulic press was set up to compress the powders into $13 \mathrm{~mm}$ diameter discs by using special forming dies. The resulting thickness was dependent on the amount of material that was required to press a pellet of the specified density. Using a precision electronic balance to accurately measure the amount of powder to use resulted in thickness accuracy's of a few percent. During the pressing, the volume between the dies was evacuated to eliminate voids in order to maintain uniformity of the pellets. Some of the pellets required the addition of a low $\mathrm{Z}$ binding material to increase their thickness to maintain structural stability. Usually, sodium acetate was used for this. Also, some of the materials used were in an oxide form to reduce water absorption. Once the pellets were complete, they were adhered to mounting rings to increase mechanical stability and make handling easier.

\section{HOUSING}

It was desirable to keep the physical size of the instrument as small as possible. The benefits of this are the ability to place multiple instruments close together, and to avoid using large sections of shielding. The resulting aluminum housing was only about five inches by five and one half inches in cross section. Since all of the major components of this device relied in some fashion on any other component, a framework to assemble and accurately locate each component was developed. The housing also served as this framework and as a vacuum vessel capable of maintaining pressure of less than $5 \times 10^{-6}$ Torr. Collimators, baffles, filters, fluorescers, the detector, and fiber optics all had to be accurately aligned to one another to allow for maximum sensitivity and to prevent any uncertainty due to misalignment. This was achieved by designing the mechanical characteristics using computer assisted drafting (CAD) techniques and specifying close tolerances for machining of the components. Utilizing "high tech" machining processes such as computerized numerical control (CNC) machines, electrical discharge machining (EDM), and laser machining provided the means to meet the required specifications. Assembly techniques to stack components on reference pins and the use of precision reference surfaces to locate components were features designed into the device and ultimately provided a high degree of accuracy. The completed assembly was then checked for proper alignment using optical techniques and then verified to some extent through $\mathrm{x}$-ray calibration. During operation, a 125 micron thick mylar window was used at the entrance end to serve as a vacuum barrier and debris shield. This window as well as all the covers and attachments were sealed with viton o-rings. 


\section{BAFFLES AND COLLIMATORS}

Located along the four meter line of sight between the source and the fluorescers were a series of collimators and defining baffles. Starting near the source was a coarse aperture $25 \mathrm{~mm}$ in diameter made of one inch thick steel. About three meters towards the fluorescers was another one inch thick plate with a $60 \mathrm{~mm}$ diameter aperture. Just at the entrance to the housing was a $12 \mathrm{~mm}$ thick kinertium (tungsten alloy) plate with sixteen collimating apertures corresponding to the sixteen channels to be observed. Finally, a series of seven baffles or defining apertures made of $.5 \mathrm{~mm}$ thick tantalum were spaced $25 \mathrm{~mm}$ apart along the remaining distance to the fluorescers. These last seven baffles were carefully designed to assure that the slightly diverging beam would only be intercepted by the individual fluorescers thus eliminating crosstalk between channels. To locate these baffles, a series of precision slots machined by wire EDM were fabricated into the housing. Each baffle could then easily be removed and accurately reinstalled. Additional slots were also provided to allow more baffles to be added.

\section{FILTER AND FLUORESCER MOUNTS}

As mentioned earlier, the fluorescer and filter pellets were adhered to stainless steel mounting rings to improve handling. These in turn were mounted into retaining frames that would capture them and provide a means to locate the entire array relative to the rest of the system. Three unique frames were built. One for the pre filters, one for the fluorescers, and one for the post filters. These frames, once assembled with their required pellets were then mounted to an "A frame" fixture using locating pins to assure accurate alignment. This mounting scheme allows the pre filters to be positioned normal to the incident $x$-rays, the fluorescers to be at $45^{\circ}$ to the incident beam and detector, and the post filter to be normal to the fluoresced $x$-rays between the detector. Each retaining frame could easily be removed to replace or exchange pellets and accurately be replaced without sacrificing alignment integrity.

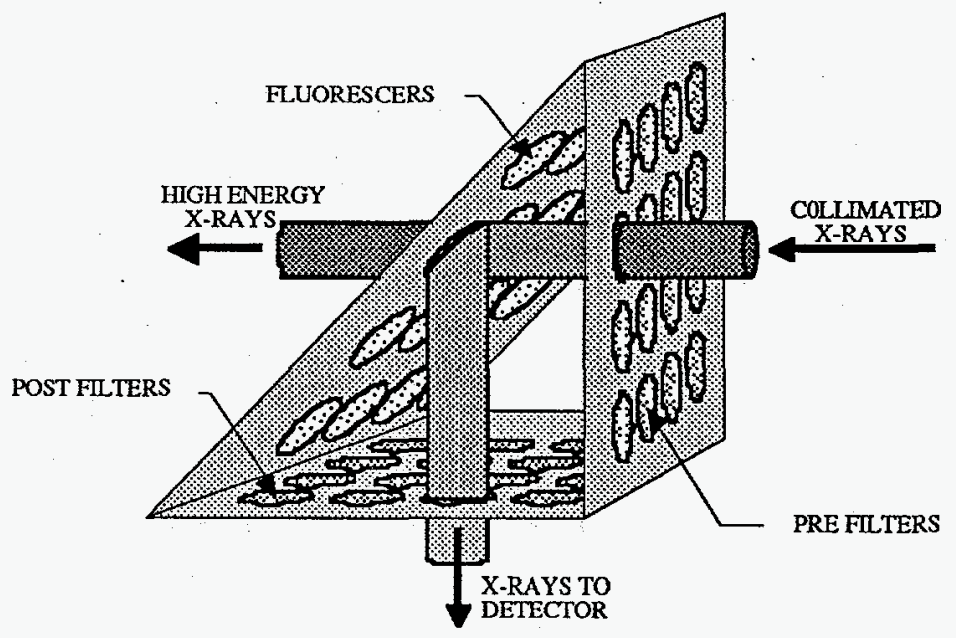

figure 3.

\section{DETECTOR TRANSITION}

As previously stated, the detector to be used already existed and therefore had some influence on how the entire instrument was built. The detector is built around a 6-3/4 inch diameter flange. Since the housing was only five inches wide at the point where the detector would mount, a cone shaped adapter was necessary. This piece also provided the means to position the detector off axis from the $\mathrm{x}$-rays incident upon the fluorescers. Inside this appendage were a series of $.5 \mathrm{~mm}$ thick tantalum baffles precisely located to define the fluoresced $x$-ray paths onto the detector. These interchangeable apertures also proved important in eliminating crosstalk between channels on the detector. Each of these were accurately aligned to each other, the fluorescer assembly, and the detector by means of dowel pins. 


\section{MICROCHANNEL PLATE DETECTOR}

The device chosen to detect the secondary $\mathrm{x}$-rays from the fluorescer was a modified microchannel plate intensified, electrostatically focusing detector that had been originally developed for the Nuclear. Test Program of years past. Incorporating some simple changes provided sixteen highly sensitive channels with the ability to vary the sensitivity in four groups of four channels each. The main components are the microchannel plate (mcp), the focusing grid, and a phosphor coated fiber optic face plate which were all installed onto a removable vacuum flange.

The mcp used is an $86 \mathrm{~mm}$ diameter image quality mcp with four gold photocathode strips deposited on the input side. Each strip can be individually biased up to - 1000 volts. This feature provides for the tuning of the detector sensitivity as required by the intensity variation of different energy channels. The back side of the mcp is coated with gold and is electrically at ground. Immediately behind the mcp lies the electrostatic focusing grid. This is a grounded stainless steel plate with a series of tapered holes that correspond to the individual beams of fluoresced $x$-rays. The tapered holes create a two dimensional compression of the electrons increasing their density by more than a factor of 100 . These focused electrons then impinge upon the aluminized cadmium sulfide phosphor coated face plate that has $\mathrm{a}+15 \mathrm{kV}$ bias on it. The green light emitted by the excitation of the phosphor is transmitted through the fiber optic face plate that serves as a vacuum window and displayed as sixteen equally spaced spots of approximately 200 microns in diameter. This system can provide a luminous gain of about 10,000. Figure 4 . describes the function of one individual channel.

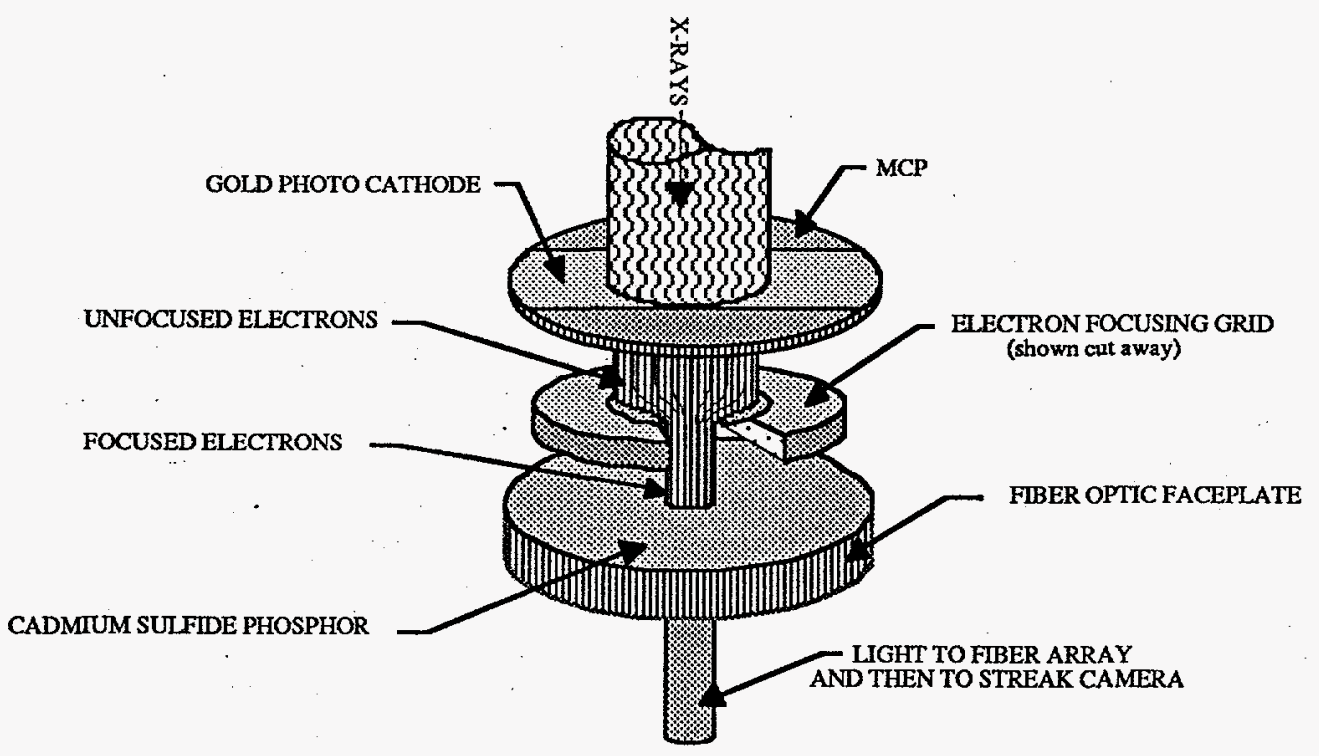

figure 4.

\section{FIBER OPTIC ARRAY}

In order to transmit the detector output to a recording device, a fiber optic array consisting of 200 micron core fibers was prepared. An individual fiber was attached to each channel output using modified SMA type connectors mounted into a fixture that could be aligned to receive all of the light signal from the 200 micron spots. Several spare fibers were also provided as backups which were also used for background signals. At the opposite end of the twelve meter long fiber bundle the fibers had previously been arranged into a row of fibers stacked and epoxied side by side and then polished flat. This linear array head could then be installed into a mounting fixture on either the time integrating camera used for calibrations or the time resolving streak camera used for the actual shots. 


\section{CALIBRATION}

Although the flourescence and transmissions of the materials used in this experiment are well understood, verification of these characteristics was necessary to decrease experimental uncertainties. Also, determining the detector response over this extreme energy range was necessary as little was known about this, especially at the higher end of the spectrum. To perform these calibrations a variable energy $\mathrm{x}$-ray fluorescence source was used in combination with well characterized detectors. A positioning device was also used to scan from channel to channel in order to expedite the calibration process.

The first step was to measure the absorption of each filter and fluorescer by subtracting the attenuated beam from the unatenuated beam at energies at, and just above the absorption edge of the particular element. A sodium iodide detector was used as a reference for these measurements. A total of sixty four data points were obtained as each pre filter and fluorescer were measured for the two devices used. Figure 5. shows a sample of the channel response curves with corresponding background channel response for a low energy and a high energy channel. The next step in the calibration process was to measure the actual fluorescense of the fluorescers. This was done without filters using a continuum source and reading the output with a germanium detector.
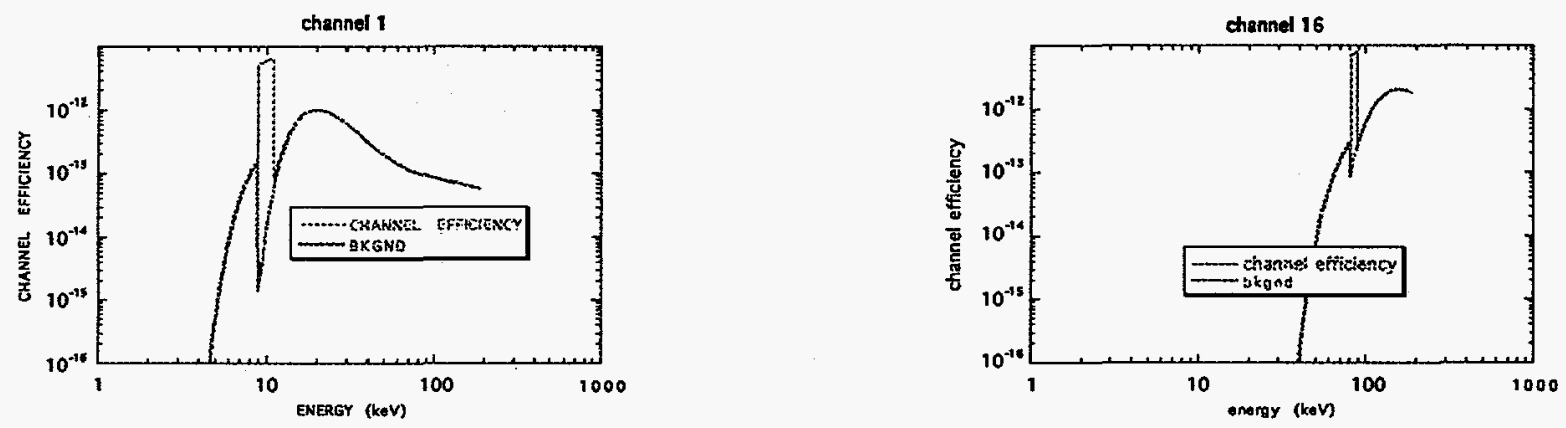

figure 5.

A determination of the detector response was also necessary as little was known about it's efficiency over such a broad spectrum, especially at higher energies. To measure each channel at each energy would be tedious and unnecessary so instead, the sensitivity of two selected channels was measured at all energies of interest. This was also done at a variety of gain settings. To obtain a relative measurement between channels, all of them were characterized at only two energies, also at increments over the entire range of gain settings. These measurements were performed using the fiber optic array connected as it would be in the actual experiment. The output was observed using an intensified reticon camera and displayed by a time integrated acquisition system. Figure 6. displays the resulting detector response for a number of channels for each detector used. It is important to notice that a slight efficiency variation between channels on the low energy detector was observed which was partially due to misalignment of the fibers. Also, a variation in the transmission of the fibers contributed to this but was not an issue since the fibers could always be positioned where they were calibrated.
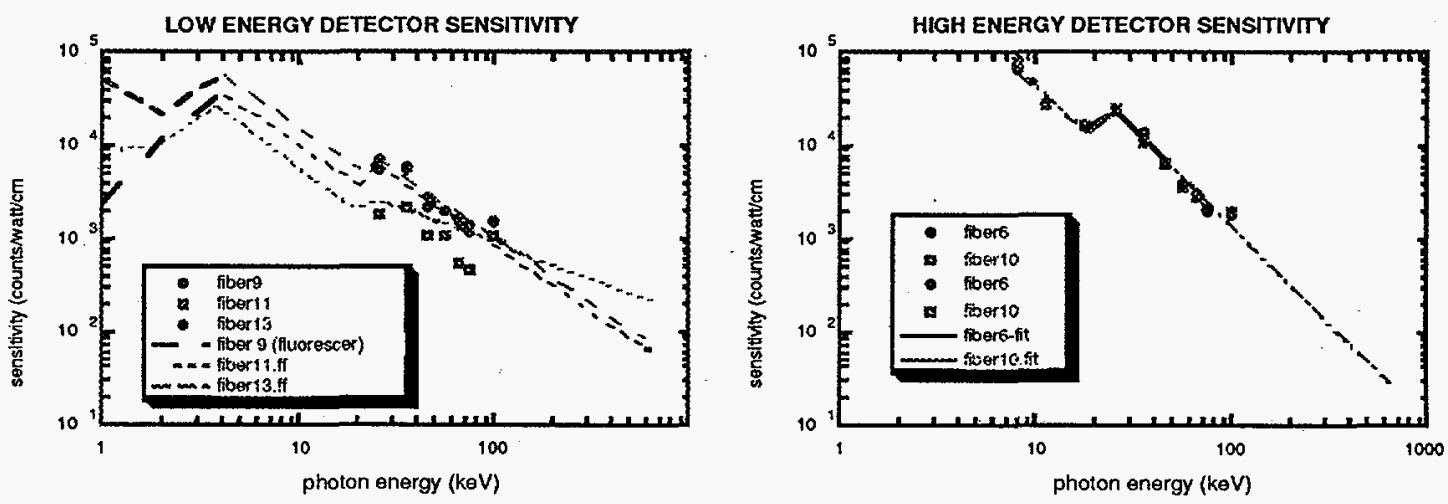

figure 6 . 


\section{INSTALLATION}

As previously mentioned, two assemblies were required in order to accommodate sixteen channels that would cover the spectral range desired with reasonable energy resolution. Figure 7. shows an end view of how the two assemblies were situated as mounted on the Saturn diagnostic port. It is worthy to note that all internal and external components were easily accessible to allow for expeditious maintenance and modification as necessary. An example of the benefit of this feature will be reviewed later. Another useful feature is that both units function independent of each other and the Saturn chamber itself. They are both vacuum isolated by means of a 250 micron thick mylar window that also serves as a debris barrier. Each one has it's own vacuum pumping system and electrical supply system which allows one system to be operational even if the other is not.

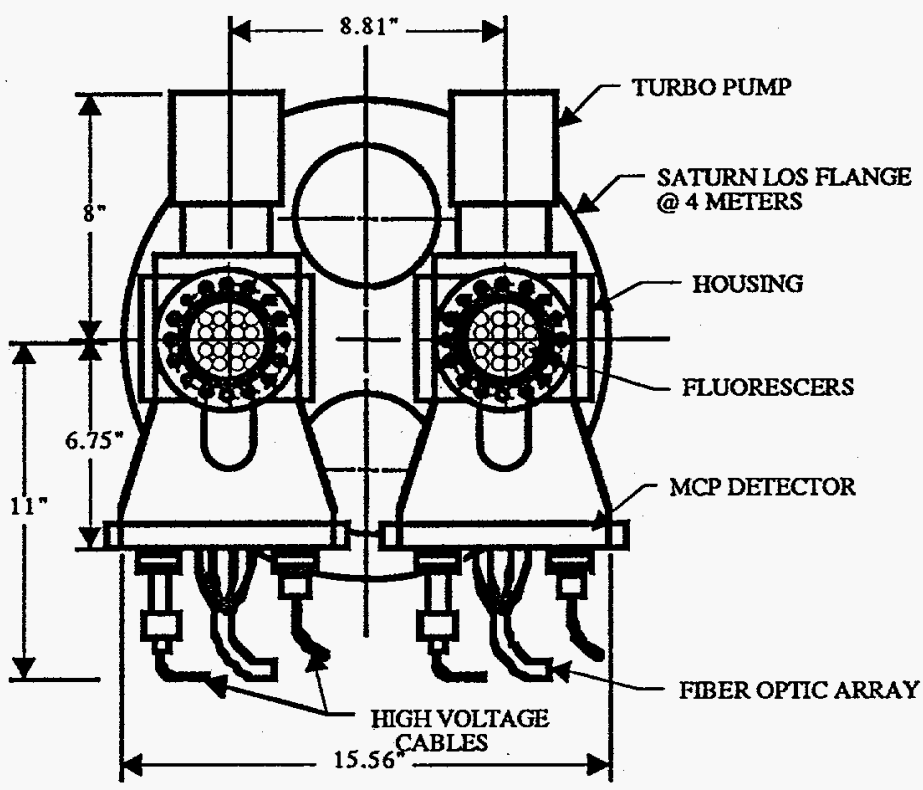

figure 7.

\section{SHIELDING}

Shielding of all of the radiation sensitive components was an issue of great importance. An abundance of hard $x$-rays and EMP fields that are not completely understood were a major concern. Any electronic devices in close proximity to the chamber area were housed in "screen boxes" or tightly sealed, well grounded enclosures to prevent instrumentation failure or alteration of data signals. Shielding from hard $\mathrm{x}$-rays was necessary to reduce potentially large background signals. Realizing where the source of this radiation was helped to determine where and how much shielding to use although utilizing as much as possible became a rule of thumb. Even though the filter/fluorescer scheme was inherently designed to provide a means to reject most backgrounds, additional care was taken to shield the detector and streak camera system. Assuming the source of most of the radiation was from the load area and diminishes over distance, lead shielding was concentrated near the most sensitive components mainly between them and the source. Steel collimators in the line of sight along with a one inch thick lead baffle just before the housings provided the bulk of the protection. Sheets of lead were used to wrap the detector in attempt to reduce scattered radiation from being detected. Lead bricks and bags of lead shot were also used to fill gaps in this "radiation umbrella". In all, a minimum of an inch of lead protected most of the device with considerably more in some areas.

\section{EXPERIMENTAL ALTERATIONS}

The versatility and interchangeability of this instrument proved to be invaluable as an unpredicted change in the experimental objectives occurred after the first few shots had been taken. Although the overall sensitivity of this device is relatively high, the $\mathbf{x}$-ray intensity was two orders of magnitude less than expected. These low signals were detectable but so weak that once the backgrounds were subtracted, the net intensity was lower than the estimated uncertainty of the device. To compensate for this problem, each unit was modified in place to provide two channels each. In one, all of the fluorescers were replaced with a large, 
single copper fluorescer, half of which was not filtered and half with a copper filter to provide a background channel. The spectral range of this channel was 9 to $20 \mathrm{keV}$. In the other unit, the fluorescers were replaced with a large, single molybdenum fluorescer with half not filtered and the other half filtered with molybdenum for the background measurement. The range of this channel was 20 to $30 \mathrm{keV}$. The channel response for these two detectors is shown in figure $7 .^{2}$ Also, all of the baffles before and after the fluorescers were replaced with "impromptu" baffles that would separate the data from background channels in each device. Removing the baffles in front of the fluorescer provided an increase in signal by enlarging the solid angle of the incident $x$-rays. As a result of these changes, eight of the channels of each detector would measure the signal while eight would measure background.
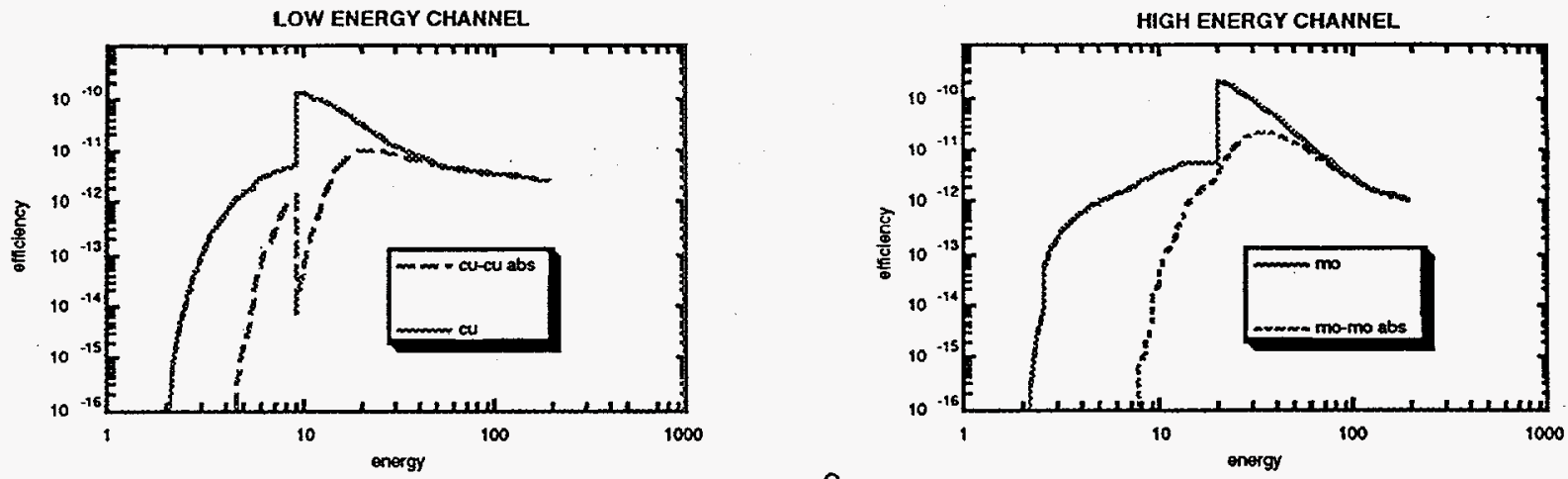

figure 8 .

\section{RESULTS OF SATURN EXPERIMENTS}

Once the modifications were made to increase the sensitivity, meaningful data could be obtained and the remainder of the experimental series could be completed. Combining the measured detector response with a calculated filter/fluorescer response allowed an overall channel response to be determined that could then be used to analyze the data acquired by each shot. Figure 8 . shows a sample of the analyzed emission data from a randomly chosen shot. ${ }^{2}$ The remaining shot series consisted of testing wire arrays of different masses. Once compiled, the data concluded that 25 Joules of radiation was produced between 10 and 100 $\mathrm{keV} .^{3}$
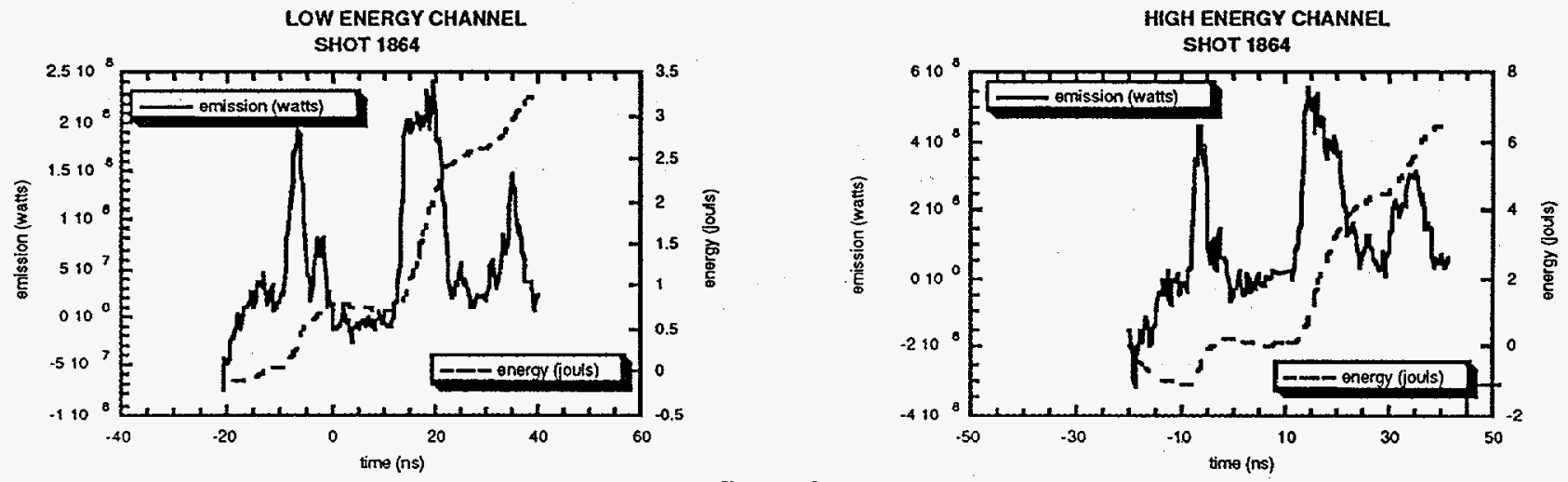

figure 9 .

\section{CONCLUSION}

The concept and design of this instrument proved to be of great value to this particular experimental series. Unfortunately, due to unexpected circumstances it was not used exactly as designed and calibrated although in it's modified version it was able to provide the data that was required. This confirms it's usefulness and versatility leading one to believe that it would be a valuable instrument for diagnosing radiation production from a wide variety of applications. 


\section{ACKNOWLEDGMENTS}

The authors wish to thank all of the participants involved in this endeavor from it's conception to completion. In particular they wish to thank Steve Stafford for his many hours of dedication. Also, appreciation is extended to Tom Sanford, the project leader, Tom Nash, Ray Mock, and the Saturn personnel that assisted in this experiment.

Work performed under the auspices of the U.S. Department of Energy by the Lawrence Livermore National Laboratory under contract W-7405-Eng-48.

\section{REFERENCES}

1. Tom Nash, "W Wires Nonthermal Run Plan", memo, May 1994

2. Bob Thoe, "Filter Fluorescer Spectrometer", internal report, March 1995

3. Tom Sanford, "Summary of the Jupiter Non-Thermal Load Program", memo, August 1994 